\title{
MiRNA-26a Contributes to the Acquisition of Malignant Behaviors of Doctaxel- Resistant Lung Adenocarcinoma Cells through Targeting EZH2
}

\author{
Jing Chen ${ }^{\mathrm{a}}$ Yuejuan $\mathrm{Xu}^{\mathrm{b}}$ Leilei Tao ${ }^{\mathrm{a}}$ Yan Pan ${ }^{\mathrm{a}}$ Kai Zhang ${ }^{\mathrm{a}}$ Rui Wang ${ }^{\mathrm{a}}$ \\ Xiaoyuan $\mathrm{Chu}^{\mathrm{a}}$ Longbang Chen ${ }^{\mathrm{a}}$ \\ aDepartment of Medical Oncology, Jinling Hospital, School of Medicine, Nanjing University, Nanjing, \\ ${ }^{\text {bDepartment }}$ of Medical Oncology, Nanjing second Hospital, Nanjing, China
}

\section{Key Words}

Lung adenocarcinoma - microRNA-26a - Enhancer of zeste homolog 2 - Epithelial-tomesenchymal $\cdot$ Chemoresistance

\begin{abstract}
Background/Aims: Accumulating evidence revealed that microRNAs (miRNAs) have been demonstrated as critical molecules in tumor development and progression. MiR-26a, located in a fragile chromosomal region associated with various human cancer, has been reported to be involved in regulating various cellular process, such as proliferation, apoptosis and invasion through targeting multiple oncogene. Docetaxel-mediated chemotherapy has been applied in improving the survival and prognosis of patients with advanced lung adenocarcinoma (LAD). However, chemoresistance remains a major impediment to clinical application of this agent. It has been presented that decreased miR-26a expression lead to cisplatin resistance and promoted growth and migration in human lung cancer. Enhancer of zeste homolog 2 (EZH2) is the target of miR-26a. The present study aimed to investigate the function of miR-26a/EZH2 in the acquisition of malignant behaviors of LAD. Methods: MiR-26a and EZH2 expression levels in the dcetaxel-insensitive groups $(n=19)$ and the docetaxel-sensitive groups $(n=18)$ were assessed by qRT-PCR. Colony formation assay, flow cytometric analysis, wound healing assay, cell transwell assays and western blotting were performed to assess the effects of miR-26a on proliferation, apoptosis and epithelial-to-mesenchymal (EMT) phenotypes in docetaxelresistant LAD cells in vitro. Xenograft transplantation, immunohistochemistry, tunel assays and western blotting assays were employed to demonstrate the role of miR-26a in docetaxelresistant LAD cells in vivo. The expression level of EZH2 in docetaxel-resistant LAD cells and corresponding parental cells was detected by qRT-PCR and western blotting. The relationship between miR-26a and EZH2 was confirmed by luciferase reporter assay. And rescue assays were performed to further confirm that miRNA-26a contributes to the acquisition of malignant behaviors of docetaxel-resistant LAD cells through targeting EZH2. Results: MiR-26a was J. Chen and Y. Xu contributed equally to this work.
\end{abstract}




\section{Cellular Physiology Cell Physiol Biochem 2017;41:583-597 and Biochemistry Publisned online: February 03, 2017 www.karger.com/cpb

significantly down-regulated in the dcetaxel-insensitive groups $(n=19)$ compared with the docetaxel-sensitive groups $(n=18)$ assessed by qRT-PCR. MiR-26a decreased the proliferation, increased the apoptosis rate and reversed EMT to MET of docetaxel-resistant LAD cells both in vivo and vitro. $\mathrm{EZH} 2$ was confirmed as target of miR-26a. Rescue assays further verified that the function of miR-26a exerts in docetaxel-resistant LAD cells is through targeting EZH2. Conclusions: Our data revealed that overexpression of miR-26a in docetaxel-resistant LAD cells could decrease the proliferation, increase the apoptosis rate and reverse EMT to MET of docetaxel-resistant LAD cells both in vivo and vitro and such function is partially exerted via downregulating EZH2. MiR-26a/EZH2 signal pathway makes contribute to the malignant phenotype of docetaxel-resistant of LAD cells which indicated that miR-26a exerts pivotal functions in the molecular etiology of chemoresistant lung adenocarcinoma.

\section{Introduction}

Non-small cell lung cancer (NSCLC), is the predominant form of lung cancer, among which, LAD is the most common histological type. LAD, always diagnosed at advanced stage, has been documented as the leading cause of cancer-related deaths [1]. Despite the development of molecular mechanisms underlying LAD has been made and treatments for LAD have been improved, the overall survival time is still limited [2]. The current therapies for advanced LAD mainly include surgery, radiotherapy, chemotherapy, local treatments and targeted therapies [3]. Docetaxel-mediated chemotherapy has been applied in improving the survival and prognosis of patients with advanced LAD. However, chemoresistance remains a major impediment to clinical application of this agent. Cells resistant to chemotherapy always exert more malignant behaviors, such as obtain a higher proliferative ability and stronger capability for invasion and metastasis. The mechanism underlying the malignant phenotype has not been fully cleared. Therefore, investigating the mechanism might be favor to explore new treatment strategies for LAD patients with chemoresistance.

MicroRNAs, a class of endogenous, small (18-25 nucleotides) non-coding RNAs, exert their functions by directly binding to the $3^{\prime}$-untranslated regions (3'UTR) of the target messenger RNAs, causing the degradation of the mRNA or translational inhibition of functional proteins $[4,5]$. Emerging evidences show that alteration of microRNAs is involved in cancer initial and progression [6-12]. MicroRNA-26a is commonly dysregulation in diverse cancers, and involves in various biological processes, including proliferation, migration, invasion, angiogenesis and metabolism, via targeting multiple mRNAs. Besides, it has been revealed that decreased miR-26a expression could cause cisplatin resistance and promote growth and migration in human lung cancer $[13,14]$. Thus, miR-26a might be involved in the malignant phenotype acquisition of docetaxel-resistant LAD cells.

Enhancer of zeste homolog 2 (EZH2) is the target of miR-26a. It has been demonstrated that miR-26a/EZH2 signal pathway contributes to the formation of EMT in HCC [15]. And down-expression of EZH2 could enhance the cisplatin-induced apoptosis in Osteosarcoma cell [16]. However, whether EZH2 contributes to the malignant phenotype acquisition of docetaxel-resistant LAD cells remains unknown. In the present study, we aimed to explore the functional role of miR-26a and EZH2 in the malignant phenotype acquisition of docetaxelresistant LAD cells.

\section{Material and Methods}

\section{Patients}

A total of 37 LAD tissues were obtained from patients diagnosed with advanced LAD in Department of Medical Oncology, Jinling Hospital (Nanjing, PR China) between March 2009 and September 2010. All the following criteria were met: a histological diagnosis of primary LAD with at least one measurable lesion; a clinical stage IIIB-IV; first-line chemotherapy with either docetaxel $75 \mathrm{mg} / \mathrm{m}^{2}$ and cisplatin $100 \mathrm{mg} / \mathrm{m}^{2}$ 


\section{Cellular Physiology Cell Physiol Biochem 2017;41:583-597 \begin{tabular}{l|l|l} 
and Biochemistry Publisned online: February 03, 2017 & $\begin{array}{l}\text { @ } 2017 \text { The Author(s). Published by S. Karger AG, Basel } \\
\text { www.karger.com/cpb }\end{array}$ \\
\hline
\end{tabular} \\ Chen et al.: MiR-26a Regulates the LAD Through EZH2}

or docetaxel $75 \mathrm{mg} / \mathrm{m}^{2}$ and carboplatin area under the curve $6 \mathrm{mg} / \mathrm{mL} / \mathrm{min}$ administered every 3 weeks for a maximum of 5 cycles. Tissue samples were divided into "sensitive" (complete or partial response) $(n=18)$ and "insensitive" $(n=19)$ (stable or progressive disease) groups based on the patient responses assessed by medical image analysis and detection of serum tumor markers after 4 or 5 cycles of docetaxelbased chemotherapy. The research protocol was reviewed and approved by the Ethical Committee and Institutional Review Board of Jinling Hospital, and written informed consent was obtained from each patient included in the study.

\section{Cell lines}

Two human LAD cell lines SPC-A1 and H1299 were purchased from the Tumor Cell Bank of Chinese Academy of Medical Science (Shanghai, China) and the docetaxel-resistant LAD cells (SPC-A1/DTX and H1299/DTX) derived from parental SPC-A1 and H1299 cells, respectively, were established and preserved in 50ug/L final concentration of docetaxel. All cells were cultured in RPMI 1640 medium containing 10\% fetal bovine serum and ampicillin and streptomycin at $37^{\circ} \mathrm{C}$ in a humidified atmosphere of $95 \%$ air and $5 \%$ $\mathrm{CO}_{2}$.

Real-time quantitative reverse-transcription polymerase chain reaction ( $q R T-P C R$ )

Total RNA from cells was isolated with Trizol reagent (Invitrogen, CA, USA) according to the manufacturer's protocol. Reverse transcription was performed with PrimeScript RT reagent Kit (Takara, Japan) according to the manufacturer's instructions. qRT-PCR was performed with SYBR Prime Script RTPCR Kits (Takara, Japan) based on the manufacturer's instructions. The miR-26a or EZH2 level was calculated with the $2^{-\Delta \Delta C t}$ method which were normalized to U6 rRNA or GAPDH mRNA, respectively. All assays were performed in triplicate. The expression levels were relative to the fold change of the corresponding controls which were defined as 1.0.

\section{Cell transfection}

Transfections with pcDNA3.1/EZH2, pcDNA3.1/miR-26a, siRNA/EZH2 (all obtained from GenePharma, Shanghai, China) and miR-26a mimics and inhibitor (all obtained from ABM, Canada) were performed using Lipofectamine 2000 (Invitrogen, USA), according to the manufacturer's protocol.

\section{Dual luciferase reporter assay}

pmirGLO, pmirGLO-EZH2-wt or pmirGLO-EZH2-mut (miR-26a) was co-transfected with miR-26a mimics or miR-NC into SPC-A1/DTX cells by Lipofectamine-mediated gene transfer. The relative luciferase activity was normalized to Renilla luciferase activity $48 \mathrm{~h}$ after transfection. The data were relative to the fold change of the corresponding control groups defined as 1.0.

\section{Colony formation assay}

Cells (500 cells / well) with or without transfection were plated in 6-well plates and incubated in RPMI 1640 with $10 \% \mathrm{FBS}$ at $37^{\circ} \mathrm{C}$. Two weeks later, the cells were fixed and stained with $0.1 \%$ crystal violet. The number of visible colonies was counted manually. All samples were assayed in triplicate.

Flow cytometric analysis of apoptosis

Apoptosis were performed using flow cytometric analyses with Annexin V: FITC Apoptosis Detection Kits (BD Biosciences, USA), according to the manufacturer's instructions. All samples were assayed in triplicate.

\section{Wound healing assays}

Cell migration capacity was calculated by wound healing assay. $2 \times 10^{5}$ cells with or without transfection were plated into 12 -well plates and incubated in RPMI 1640 with $10 \% \mathrm{FBS}$ at $37^{\circ} \mathrm{C}$. After reaching $100 \%$ confluence, cells were wounded by scraping with a $200 \mu \mathrm{l}$ tip, following washed 3 times in serum-free medium and incubated in regular medium. Wounds were observed at 0 and $48 \mathrm{~h}$. The cell migration distance was calculated by subtracting the wound width at each time point from the wound width at the $0 \mathrm{~h}$ time point. Three independent assays were assayed. 


\section{Cellular Physiology Cell Physiol Biochem 2017;41:583-597 and Biochemistry Published online: February 03, 2017 www.karger.com/cpb \\ Chen et al:: MiR-26a Regulates the LAD Through EZH2}

Cell migration and invasion assays

Cell migration and invasion were measured by transwell chamber (8um pore size, Corning) and Matrigel invasion (Bection Dickinson), respectively. $48 \mathrm{~h}$ after transfection, cells in serum-free media were placed into the upper chamber coated with or without $10 \mathrm{ug} / \mathrm{ml}$ Matrigel. Media containing 10\% FBS were added into the lower chamber. Following $48 \mathrm{~h}$ incubation, cells remained in upper membrane were wiped, while cells migrated or invaded were fixed in methanol, stained with $0.1 \%$ crystal violet and counted under a microscope. Three independent experiments were carried out.

Western bolt analysis and antibodies

Total protein lysates were separated in $\% 10$ sodium dodecyl suifate-polyacrylamide gel electrophoresis (SDS-PAGE), and were electrophoretically transferred to polyvinylidene difluoride membranes (Roche). Protein loading was estimated using mouse anti-GAPDH monoclonal antibody. The membranes were blotted with $10 \%$ non-fat milk in TBST for $2 \mathrm{~h}$ at room temperature, washed and then probed with the rabbit antihuman E-cadherin (1:2000 dilution), $\beta$-catenin (1:2000 dilution), $\mathrm{N}$-cadherin (1:2000 dilution), vimentin (1:2000 dilution), EZH2 (1:2000 dilution), activated caspase-3 (1:2000 dilution), total caspase-3(1: 2000 dilution), and GAPDH (1:3000 dilution) overnight at $4^{\circ} \mathrm{C}$, followed by treatment with secondary antibody conjugated to horseradish peroxidase for $2 \mathrm{~h}$ at room temperature. The proteins were detected by the enhanced chemiluminescence system and exposed to x-ray film. All antibodies were purchased from Abcam (USA).

Xenograft transplantation and immunohistochemistry

Approximately $5.0 \times 10^{6}$ stably transfected with pcDNA3.1/miR-NC, pcDNA3.1/miR-26a co-transfected with pcDNA3.1 or miR-26a co-transfected with pcDNA3.1/EZH2 in SPC-A1/DTX cells suspended in 100ul PBS were injected subcutaneously into the right side of the posterior flank of female BALB/c athymic nude mice (Department of Comparative Medicine, Jinling Hospital) at 5 to 6 weeks of age. Tumor growth was examined every other day with a vernier caliper. Tumor volumes were calculated by using the equation: $\mathrm{V}=\mathrm{A}^{*} \mathrm{~B}^{2} / 2\left(\mathrm{~mm}^{3}\right)$, wherein $\mathrm{A}$ is the largest diameter and $\mathrm{B}$ is the perpendicular diameter. After 5 weeks, all mice were killed and necropsies were carried out. The primary tumors were excised, paraffin-embedded, formalin-fixed, and conducted hematoxylin and eosin (H\&E) staining, immunostaining analysis for Ki-67 and proliferating cell nuclear antigen (PCNA) protein expression according to the manufacturer's instructions. All animal experiments has been strictly conducted in accordance with the ethical standards and performed in accordance with institutional guidelines.

\section{TUNEL assay}

Apoptosis in transplanted-tumor tissues was detected by TUNEL method. The TUNEL assay was performed according to the guidelines recommended by the TUNEL assay kit (KeyGen, Nanjing, China).

\section{Statistical analysis}

Data are shown as the means \pm standard error of at least three independent experiments. The SPSS 17.0 software (SPSS Inc., Chicago, IL, USA) was used for statistical analysis. Two group comparisons were performed with a Student t test. Multiple group comparisons were analyzed with one-way ANOVA. All tests performed were two-sided. Statistically significant positive correlation between miR-26a and EZH2 expression levels in 37 cases of LAD tissues was analyzed by Spearman's correlation analysis. $\mathrm{P}<0.05$ was considered statistically significant.

\section{Results}

MiR-26a is down-expression in human docetaxel-insensitive LAD tissues and docetaxelresistant cell lines

A total of 37 cases of clinical LAD tissues were obtained from patients at advanced stage and divided into "sensitive" (complete or partial response) and "insensitive" (stable or progressive disease) groups in response to the docetaxel-based chemotherapies. MiR-26a was significantly down-regulated in the dcetaxel-insensitive groups $(n=19)$ compared with 


\section{Cellular Physiology \\ Cell Physiol Biochem 2017;41:583-597

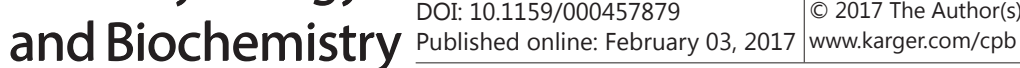

Fig. 1. MiR-26a is down-expression in human docetaxel-insensitive LAD tissues and docetaxel-resistant cell lines. (A) MiR-26a was significantly down-regulated in the dcetaxel-insensitive groups $(\mathrm{n}=19)$ compared with the docetaxel-sensitive groups ( $\mathrm{n}=18)$ assessed by qRT-PCR. (B) Difference in miR-26a expression levels C between docetaxel-resistant LAD cells and corresponding parental cells. (C) The relative level of EZH2 expression in the "insensitive" ( $\mathrm{n}=19$ ) and the "sensitive" groups (n = 18). (D) A statistically significant negative correlation

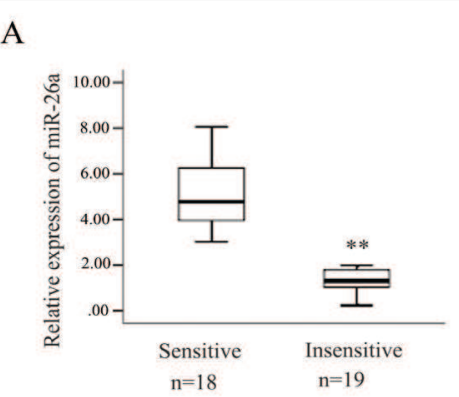

B
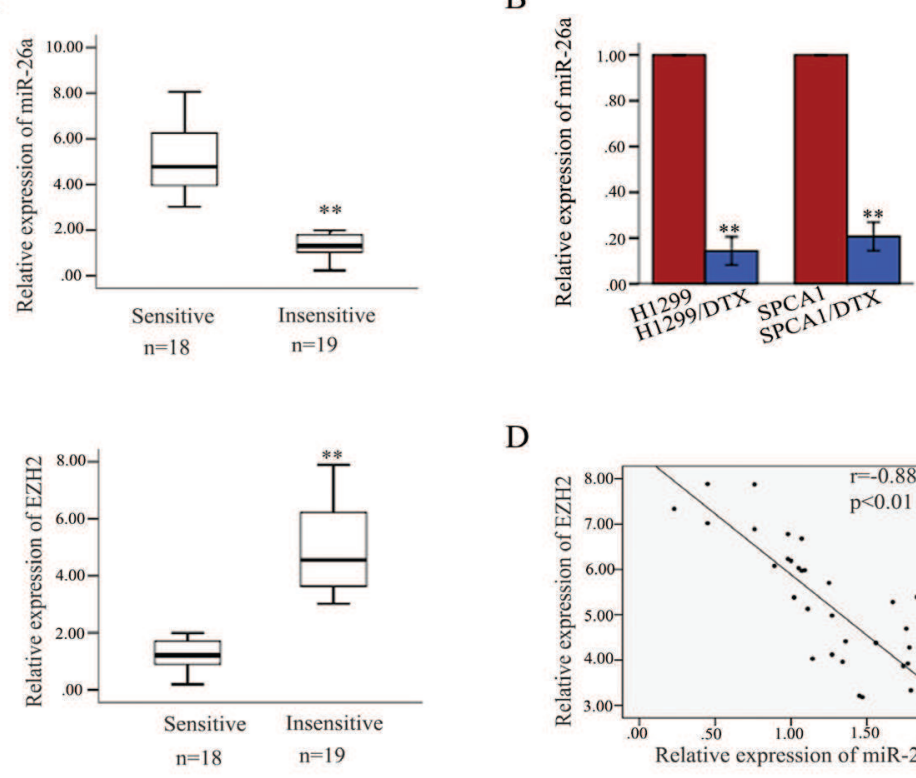

D

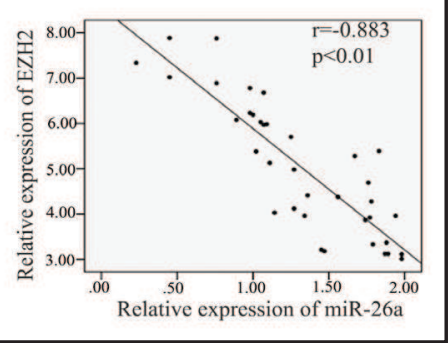

between miR-26a and EZH2 expression levels in 37cases of LAD tissues (Spearman's correlation analysis, $r=-0.883 ; P<0.01$ ).Error bars represent the mean \pm SEM of at least three independent experiments. ${ }^{*} p<$ $0.05, * * \mathrm{p}<0.01$ vs. control group.

the docetaxel-sensitive groups $(n=18)$ assessed by qRT-PCR (Fig. $1 \mathrm{~A}, \mathrm{p}<0.01$ ). Then, we examined the level of miR-26a in two docetaxel-resistant LAD cell lines (SPC-A1/DTX and H1299/DTX) and the corresponding parental cell lines (SPC-A1 and H1299). As presented in Fig. 1B, decreased expression of miR-26a was observed in docetaxel-resistant LAD cell lines compared with the corresponding parental cell lines. As a target of miR-26a, we also detected the level of EZH2 in clinical LAD tissues. As shown in Fig. 1C, the relative level of EZH2 expression in the "insensitive" ( $n=19)$ was significantly higher than that in the "sensitive" groups $(\mathrm{n}=18)(\mathrm{p}<0.01)$. Moreover, there was a significant negative correlation between EZH2 expression level and miR-26a expression level (2-tailed Spearman's correlation, $r=-0.883, p<0.01$; Fig. 1D). The result indicated that miR-26a could be involved in the malignant phenotype of docetaxel-resistant LAD cells and might through targeting EZH2.

MiR-26a is associated with proliferation, apoptosis, cell cycle and EMT of LAD cells

To investigate the biological functions of miR-26a on proliferation, apoptosis and EMT of LAD cells, H1299 (and SPC-A1) cells or H1299/DTX (and SPC-A1/DTX) cells were transfected with mirR-26a inhibitor or miR-26a mimics, respectively, using anti-miR-NC or miR-NC as a negative control (NC). Satisfactory transfection efficiency was obtained at 48 hours post-transfection (Fig. 2A and B). As shown in Fig. 2C, colony formation assay revealed down-regulated of miR-26a in H1299 and SPC-A1 cells increased the colony formation rate. While, weaken proliferation ability were observed in miR-26a transfected H1299/DTX and SPC-A1/DTX cells compared with miR-NC-transfected cells (Fig. 2D). Next, flow cytometric analysis of apoptosis was performed to detect the function of miR-26a on apoptosis in LAD cells. As presented in Fig. 2E, deletion of miR-26a significantly decreased the apoptosis rate of H1299 and SPC-A1 cells. On the contrary, compared with negative controls, forced expression of miR-26a caused an obviously increase in apoptosis rate in H1299/DTX or SPC-A1/DTX cells (Fig. 2F). Cell cycle analysis revealed that overexpression of miR-26a lead to the G2/M cell cycle arrest (Fig. 2G and H). Moreover, wound healing assays presented an increased migratory capacity of H1299 and SPC-A1 cells transfected with miR-26a inhibitor, and decreased migratory potential was observed in H1299/DTX and SPC-A1/DTX cells transfected with miR-26a mimics in comparison with negative controls (Fig. $3 \mathrm{~A}$ and B). And 


\section{Cellular Physiology \\ Cell Physiol Biochem 2017;41:583-597 \\ \begin{tabular}{ll|l} 
and Biochemistry & DOblished online: February 03, 2017 & $\begin{array}{l}\text { @ } 2017 \text { The Author(s). Published by S. Karger AG, Basel } \\
\text { www.karger.com/cpb }\end{array}$ \\
\hline
\end{tabular} \\ Chen et al.: MiR-26a Regulates the LAD Through EZH2}

A

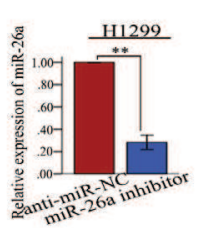

C

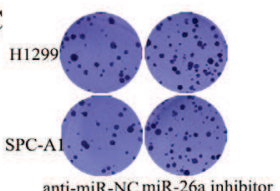

E

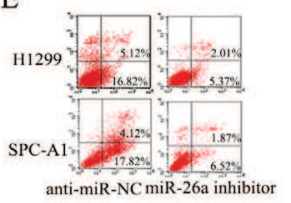

G
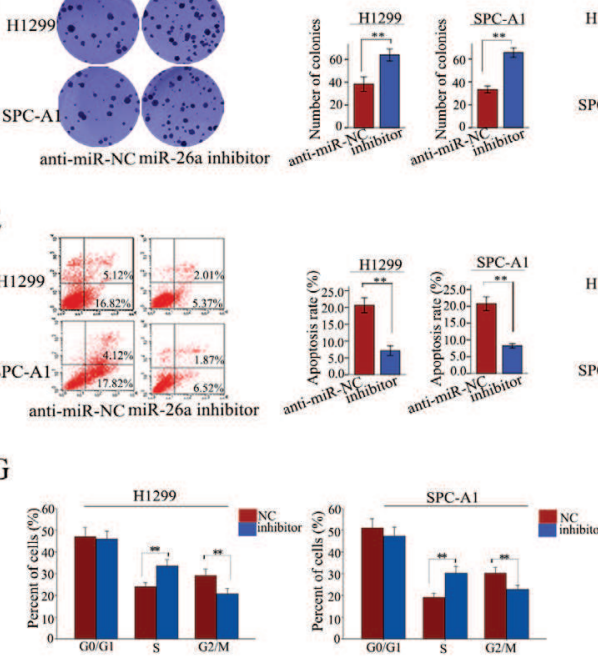

B
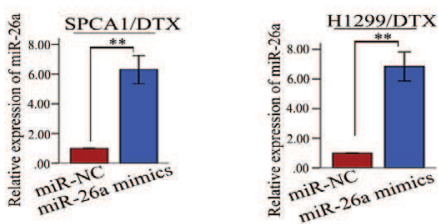

D
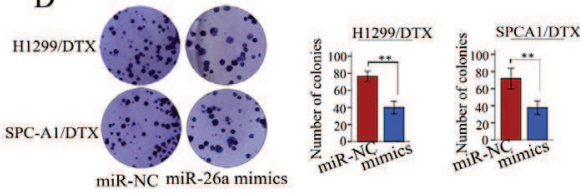

$\mathrm{F}$
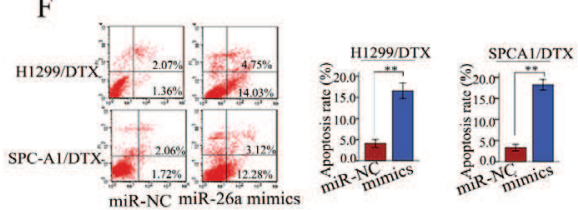

$\mathrm{H}$

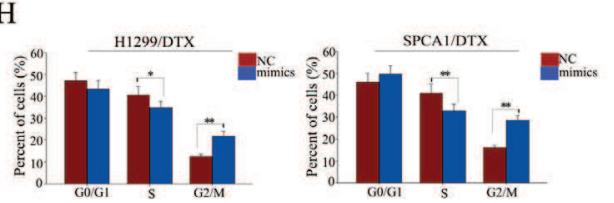

Fig. 2. MiR-26a is associated with proliferation, apoptosis and cell cycle of LAD cells (A-B) Satisfactory transfection efficiency was obtained at 48 hours after H1299 (and SPC-A1) cells or H1299/DTX (and SPC-A1/DTX cells) transfected with miR-26a inhibitor or miR-26a mimics. (C-D) Colony formation assay was performed to analyze the expression of miR-26a on colony formation capacity of docetaxel-resistant LAD cells and corresponding parental cells. (E-F) Flow cytometric analysis was employed to detect the function of miR-26a on apoptosis rate of docetaxel-resistant LAD cells and corresponding parental cells. (G-H) Cell cycle analysis was applied to exam the function of miR-26a on cell cycle of docetaxel-resistant LAD cells and corresponding parental cells. Error bars represent the mean \pm SEM of at least three independent experiments. ${ }^{*}$ p < $0.05,{ }^{* *} \mathrm{p}<0.01$ vs. control group.

migration/invasion assays were conducted to measure the metastasis/invasion capacity, as shown in Fig. 3C and D, compared with negative controls, cells transfected with miR-26a inhibitors/ mimics exert an obviously enhanced/weaken metastasis/ invasion capacity. Additionally, results from qRT-PCR and western blotting revealed that miR-26a inhibitors contributed to the formation of EMT in H1299 and SPC-A1 cells, while miR-26a mimics could significant reverse EMT to MET of docetaxel-resistant LAD cells (Fig. 3E and F). These data together indicates that miR-26a is associated with proliferation, apoptosis, cell cycle, metastasis and EMT of docetaxel-resistant LAD cells.

EZH2 is a downstream target of miR-26a and is negatively associated with miR-26a expression

Overexpression of EZH2 has been found to be associated with tumor aggressiveness, metastasis, and poor prognosis in multiple cancers [17-20], including lung cancer [21,22]. MiR-26a was documented to suppress tumor growth by targeting EZH2 in some tumors [2325]. Therefore, we hypothesized that the functions of miR-26a in docetaxel-resistant LAD cells might be mediated by EZH2. To test this assumption, we first examined the expression level of EZH2 in H1299/DTX (or SPC-A1/DTX) cells and corresponding parental cells. As illustrated in Fig. 4A, in H1299/DTX or SPC-A1/DTX cells, the expression of EZH2 both in mRNA and protein level was significantly higher than that in the parental cells. To determine 


\section{Cellular Physiology Cell Physiol Biochem 2017;41:583-597 and Biochemistry \begin{tabular}{l|l} 
DOI: 10.1159/000457879 & ( 2017 The Author(s). Published by S. Karger AG, Basel
\end{tabular}

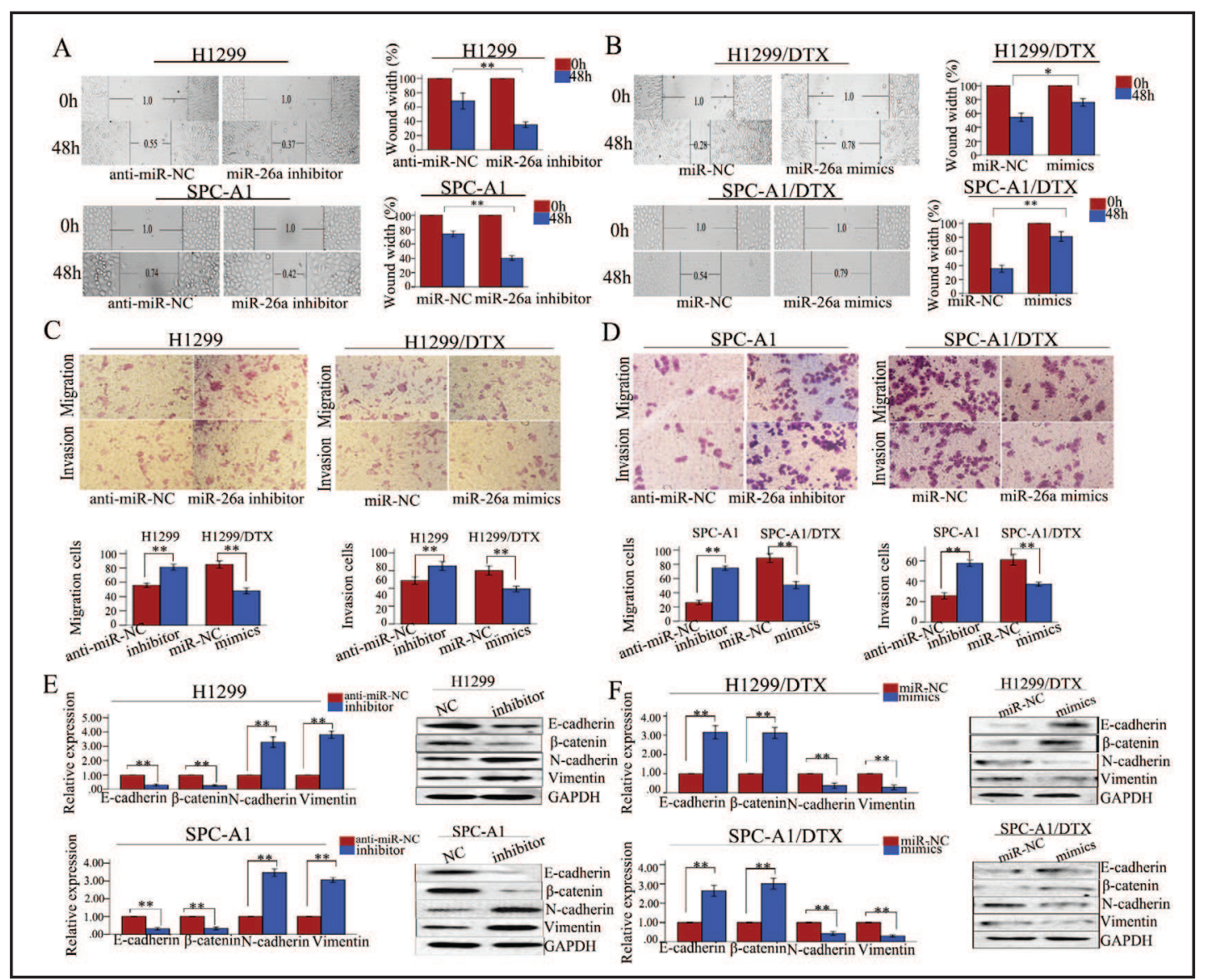

Fig. 3. MiR-26a is associated with cell migration and could reverse EMT to MET of LAD cells (A-D) Wound healing assays and transwell assays were utilized to exam the function of miR-26a on metastasis and invasion ability of docetaxel-resistant LAD cells and corresponding parental cells. (E-F) qRT-PCR and western blotting assays were performed to detect the change of EMT markers in miR-26a inhibitor transfected H1299 (and SPC-A1) cells or miR-26a transfected H1299/DTX (and SPC-A1/DTX) cells. Error bars represent the mean \pm SEM of at least three independent experiments. ${ }^{*} \mathrm{p}<0.05,{ }^{* *} \mathrm{p}<0.01$ vs. control group.

the relationship between miR-26a and EZH2, we examined the expression level of EZH2 in response to dysregulated miR-26a expression in H1299/DTX and SPC-A1/DTX cells and its corresponding parental cells. As shown in Fig. 4B, the expression of EZH2 was negatively correlated with that of miR-26a, and up-regulated miR-26a could reduce the expression of EZH2 in protein level. To further validate the regulatory relationship between miR-26a and EZH2, we performed luciferase reporter assays. As shown in Fig. 4C, miR-26a mimics reduced the luciferase activity of wild-type (WT) EZH2 reporter vector but not that of empty vector and mutant reporter vector. Together, these data suggest that EZH2 is a bona fide miR26a-targeting gene.

EZH2 is associated with proliferation, apoptosis, cell cycle and EMT of LAD cells

Due to the previously reported facilitates proliferation, inhibits apoptosis and down-regulates E-cadherin expression of EZH2 [25-28], we transfected our two parental cells (H1299 and SPC-A1)with pcDNA3.1/EZH2 and two docetaxel-resistant LAD cells (H1299/DTX, SPC-A1/DTX) with EZH2 small interfering RNA (siRNA) to explore whether dysregulated EZH2 could influence the cell proliferation, apoptosis and the formation of EMT. Satisfactory transfection efficiency was obtained at 48 hours post-transfection (Fig. 5A and B). As present in Fig. 5C, forced expression of EZH2 could increase the cell proliferation ability, compared with the controls. On the contrary, deletion of EZH2 significantly inhibited 


\section{Cellular Physiology \\ Cell Physiol Biochem 2017;41:583-597 and Biochemistry Published onlne: February 03, 2017 www.karger.com/cpb

A
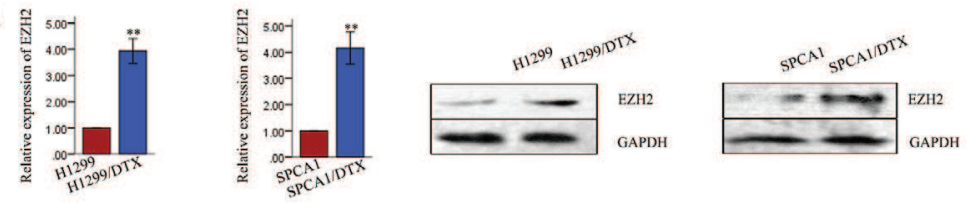

B
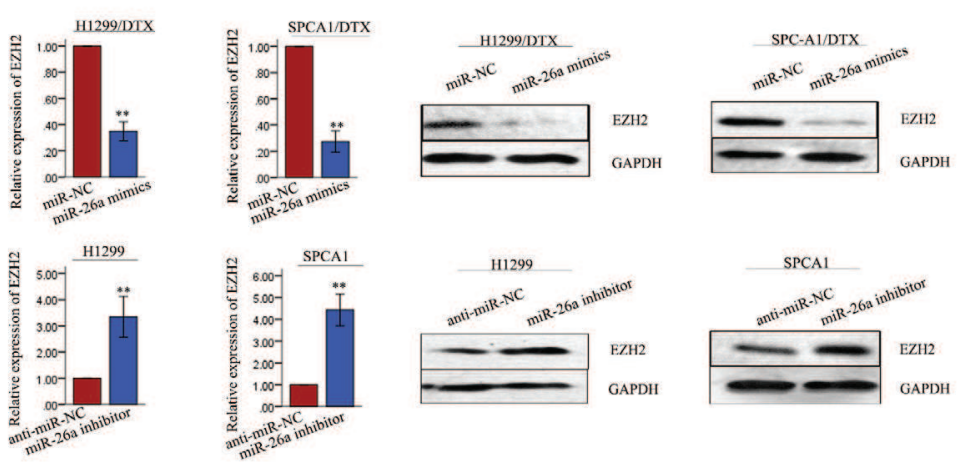

$\mathrm{C}$

hsa-miR-26a 3'-UCGgAUAGGACCUAAUGAACUU-5' WT EZH2 3'UTR 5'- CTTTGAATAAAGAATACTTGAA-3'

hsa-miR-26a 3'-UCGGAUAGgACCUAAUGAACUU-5' MUT EZH2 3'UTR 5'- CTTTGAATAAAGAAATGAACTI-3'

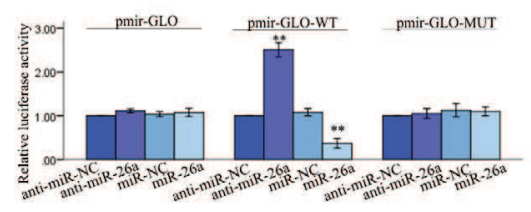

Fig. 4. EZH2 is a downstream target of miR-26a and is negatively associated with miR-26a expression. (A) qRT-PCR and western blotting were performed to detect the expression of EZH2 both in mRNA and protein level in docetaxel-resistant LAD cells and the parental cells. (B) qRT-PCR and western blotting were employed to exam the expression level of EZH2 in response to dysregulated miR-26a expression in H1299/DTX and SPC-A1/DTX cells and its corresponding parental cells. (C) Luciferase reporter assays we performed to validate the regulatory relationship between miR-26a and EZH2. Error bars represent the mean \pm SEM of at least three independent experiments. ${ }^{*} p<0.05,{ }^{* *} p<0.01$ vs. control group.

the proliferation ability of docetaxel-resistant LAD cells (Fig. 5D). Besides, results from flow cytometric analysis of apoptosis showed that overexpression of EZH2 suppressed while downexpression of EZH2 facilitated the apoptosis rate in comparison with the controls (Fig. 5E and F). Additionally, cell cycle analysis revealed that deletion of EZH2 could cause G2/M phase arrest (Fig. 5G and H). What's more, wound and transwell assays revealed that enhanced/weaken migratory capacity was observed in cells transfected with pcDNA3.1/ EZH2 or EZH2 siRNA, compared with controls (Fig. 6A-D). Results from qRT-PCR and western blot confirmed that forced expression of EZH2 contributed to the formation of EMT in parental cells while down-regulated EZH2 could reverse EMT to MET in docetacelresistant LAD cells (Fig. 6E, F).

The above results speculate that EZH2 might positively regulate proliferation, apoptosis, cell cycle, migratory and EMT of AD cells, which might be involved in the function of miR-26a in LAD.

The function of miR-26a in docetaxel-resistant LAD cells is in an EZH2-dependent manner

We performed rescue experiments to determine whether miR-26a influenced docetaxelresistant LAD cells proliferation, apoptosis, and EMT in an EZH2-dependent manner. MiR-26a mimics or miR-NC were transfected into H1299/DTX and SPC-A1/DTX cells co- transfected 


\section{Cellular Physiology \\ Cell Physiol Biochem 2017;41:583-597 and Biochemistry Published onlne: February 03, 2017 (2017ww.karger.com/cpb

A

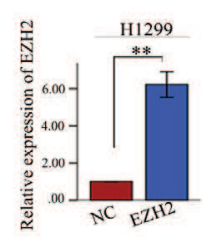

C

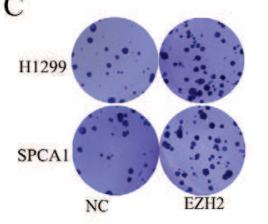

E

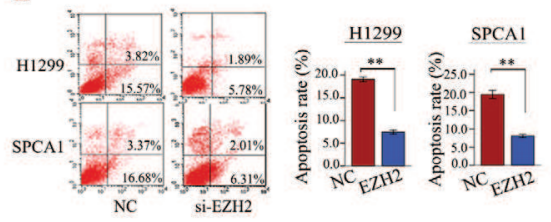

G

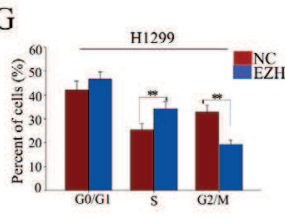

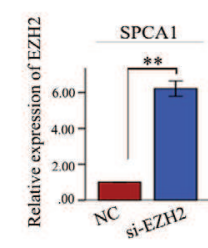

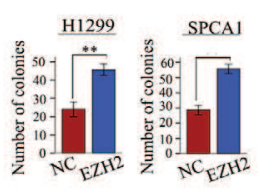

B
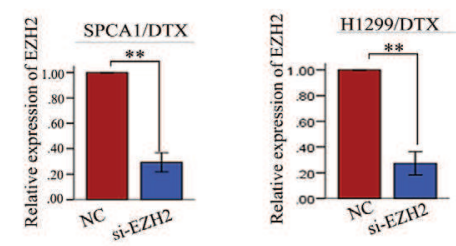

D

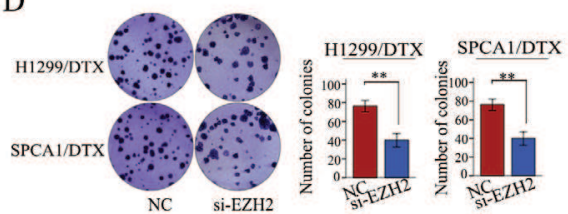

F

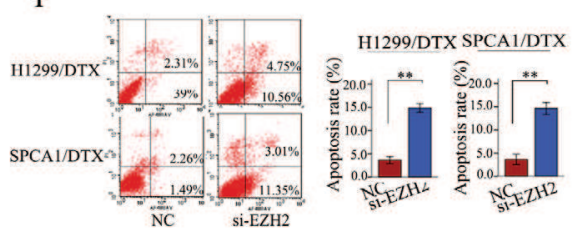

$\mathrm{H}$

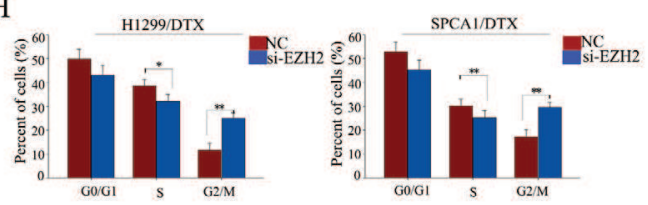

Fig. 5. EZH2 is associated with proliferation, apoptosis and cell cycle of LAD cells. (A-B) Satisfactory transfection efficiency was obtained at 48 hours after H1299 (and SPC-A1) cells or H1299/DTX (and SPC-A1/ DTX cells) transfected with pcDNA3.1/EZH2 or si-EZH2. (C-D) Colony formation assay was performed to analyze the expression of EZH2 on colony formation capacity of docetaxel-resistant LAD cells and corresponding parental cells. (E-F) Flow cytometric analysis was employed to detect the function of EZH2 on apoptosis rate of docetaxel-resistant LAD cells and corresponding parental cells. (G-H) Cell cycle analysis was applied to exam the function of EZH2 on cell cycle of docetaxel-resistant LAD cells and corresponding parental cells. Error bars represent the mean \pm SEM of at least three independent experiments. ${ }^{*} p<0.05,{ }^{* *} p$ $<0.01$ vs. control group.

with pcDNA3.1-EZH2 or pcDNA3.1. The decreased colony formation capacity induced by miR-26a in H1299 /DTX and SPC-A1/DTX cells was abrogated by the introduction of EZH2 $(\mathrm{p}<0.01$; Fig. 7A). Flow cytometric assays revealed that the proapoptotic effect of miR-26a could be partially reversed by the introduction of EZH2 ( $p<0.01$; Fig. 7B). Cell cycle analysis revealed that the G2/M phrase arrest caused by miR-26a could be partially rescued by the introduction of EZH2 (Fig. 7C). The anti-metastasis effect of miR-26a in H1299 /DTX and SPC-A1/DTX could be partly abolished by co-transfection with EZH2 $(\mathrm{p}<0.01$; Fig. 7D and E). Additionally, qRT-PCR and western blot assays indicated that the MET effect of miR-26a could be partly abrogated by EZH2 in H1299/DTX and SPC-A1/DTX cells ( $p<0.01$; Fig. 7F). These results showed that miR-26a could influence cell proliferation, apoptosis, and reverse EMT to MET phenotype in docetaxel-resisitant LAD cells in vitro at least in part in an EZH2dependent manner.

MiR-26a/EZH2-mediated signal pathway inhibited tumor growth and EMT in vivo

To explore the role of miR-26a in tumor growth and metastasis, we inoculated nude mice with SPC-A1/DTX cells stably transfected with pcDNA3.1/miR-NC, pcDNA3.1/miR26a co-transfected with pcDNA3.1 or miR-26a co-transfected with pcDNA3.1/EZH2 in 


\section{Cellular Physiology Cell Physiol Biochem 2017;41:583-597 and Biochemistry POI: 10.1159/000457879 2017 (0) 2017 The Author(s). Published by S. Karger AG, Basel}

Chen et al.: MiR-26a Regulates the LAD Through EZH2

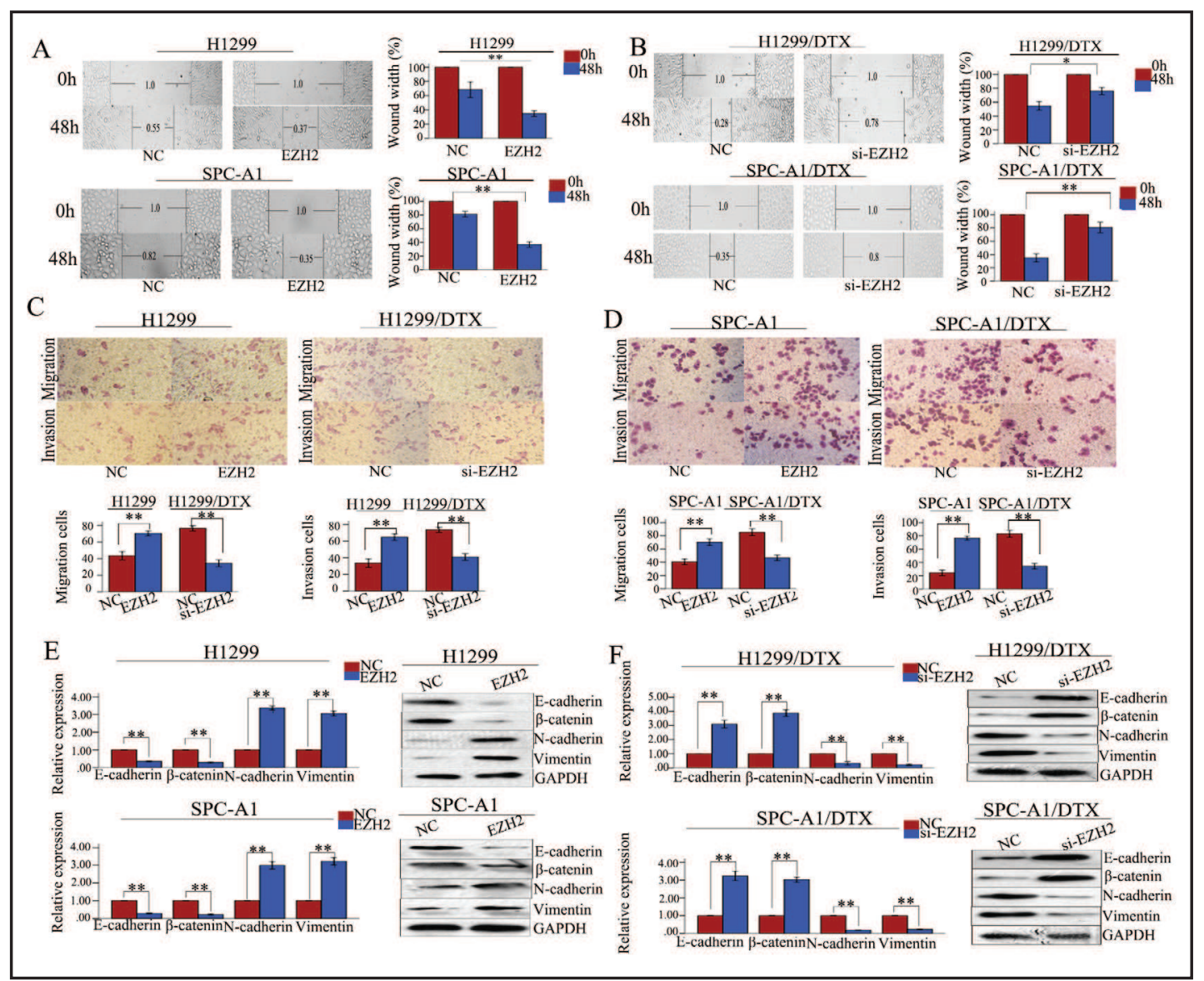

Fig. 6. EZH2 is associated with cell migration and could reverse EMT to MET of LAD cells. (A-D) Wound healing assays and transwell assays were utilized to exam the function of EZH2 on metastasis and invasion ability of docetaxel-resistant LAD cells and corresponding parental cells. (E-F) qRT-PCR and western blotting assays were performed to detect the change of EMT markers in EZH2 transfected H1299 (and SPC-A1) cells or si-EZH2 transfected H1299/DTX (and SPC-A1/DTX) cells. Error bars represent the mean \pm SEM of at least three independent experiments. ${ }^{*} \mathrm{p}<0.05,{ }^{* *} \mathrm{p}<0.01$ vs. control group.

SPC-A1/DTX. Tumors derived from pcDNA3.1/ miR-26a transfected SPC-A1/DTX cells grew more slowly than those derived from pcDNA3.1/miR-NC transfected cells, and EZH2 could partially reverse the function exerted by miR-26a (Fig. 8A). As shown in Fig. 8B, the level of epithelial protein markers was significantly increased, while expression of mesenchymal markers was obviously decreased, compared with SPC-A1/DTX / miR-NC, and such function could be partially reversed by EZH2. Immunostaining analysis revealed a lower positive rate of proliferating cell nuclear antigen (PCNA) and Ki67 (Fig. 8C). TUNEL staining and western blotting assays present a higher apoptotic rate in tumors derived from pcDNA3.1/ miR26a transfected docetaxel-resistant LAD cells compared with the control groups (Fig. 8D and E), and such phenomenon could also be partially abolished by EZH2. Together, these data suggest that miR-26a/EZH2 signaling pathway might be associated with the malignant phenotype of docetaxel-resistant LAD cells.

\section{Discussion}

Chemotherapy is a significant component of current first-line treatment for LAD patients. However, chemoresistant remains main obstacle in clinical application of this 


\section{Cellular Physiology Cell Physiol Biochem 2017;41:583-597

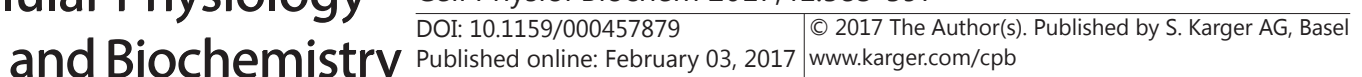

Chen et al.: MiR-26a Regulates the LAD Through EZH2

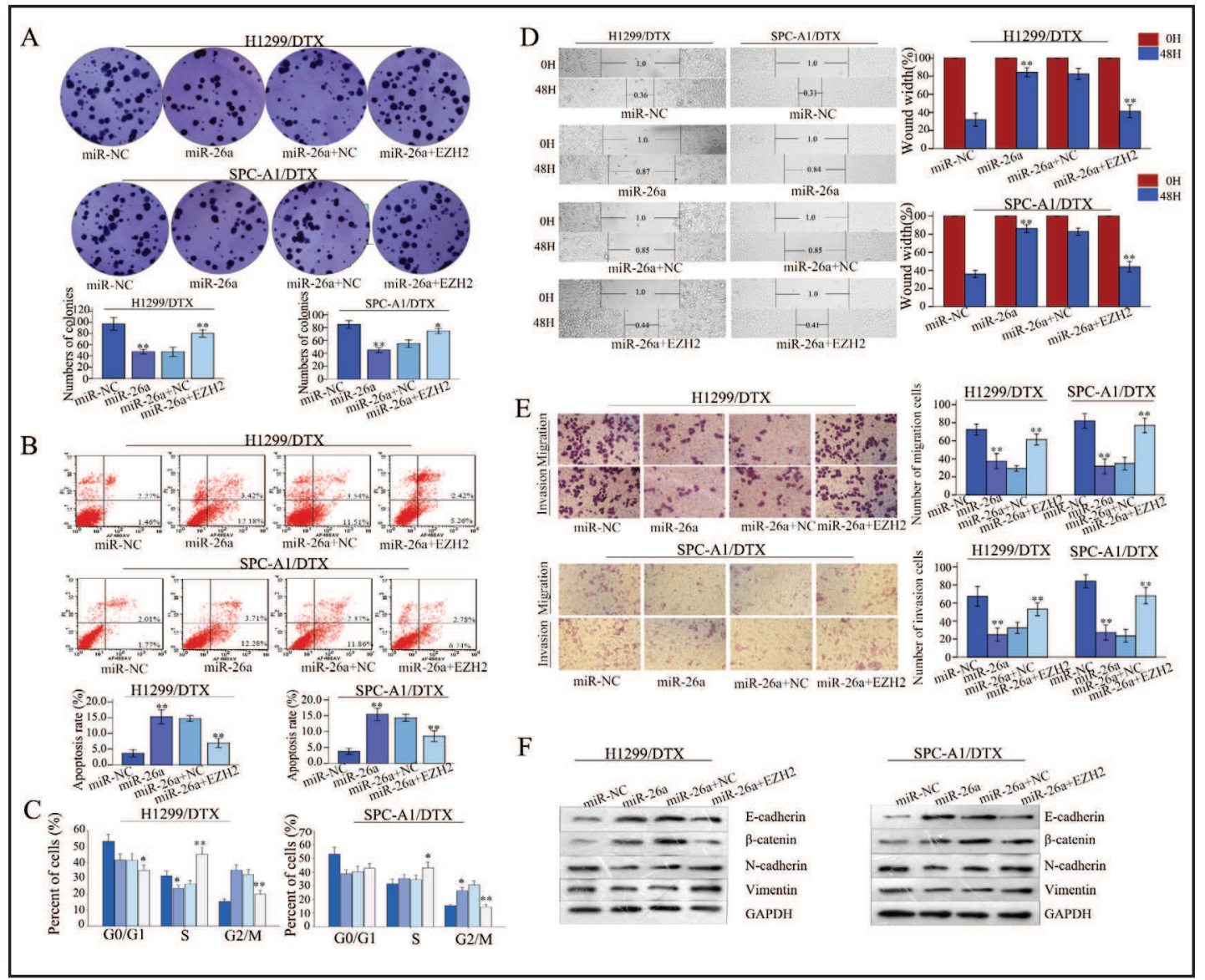

Fig. 7. The function of miR-26a in docetaxel-resistant LAD cells is in an EZH2-dependent manner. (A) The decreased colony formation capacity induced by miR-26a in H1299 /DTX and SPC-A1/DTX cells was abrogated by the introduction of EZH2. (B) Flow cytometric assays revealed that the proapoptotic effect of miR-26a could be partially reversed by the introduction of EZH2. (C) G2/M phrase arrest caused by miR26a could be partially reversed by EZH2. (D-E) The anti-metastasis effect of miR-26a in H1299 /DTX and SPC-A1/DTX could be partly abolished by co-transfection with EZH2. (F) qRT-PCR and western blot assays indicated that the MET effect of miR-26a could be partly abrogated by EZH2 in H1299/DTX and SPC-A1/ DTX cells. Error bars represent the mean \pm SEM of at least three independent experiments. ${ }^{*} \mathrm{p}<0.05,{ }^{* *} \mathrm{p}<$ 0.01 vs. control group.

agent. Cells resistant to chemotherapy always obtain enhanced proliferation ability and stronger metastasis capacity. Docetaxle-resistant LAD cells (H1299/DTX and SPC-A1/DTX) established previously by our lab also presents a higher degree malignant than the parental cells. Investigating the underlying mechanism of this phenomenon is essential for clinical treatment of LAD patients.

MiRNAs composed of 20-22 non-coding nucleotides are associated with multiple cellular processes and diseases by directly binding to the 3'-UTR of the target mRNAs. Abnormal expression of miRNA has been reported in many cancers, for instance, let-7c function as a metastasis suppressor in colorectal cancer by targeting MMP11 and PBX3, and miR-29 can regulate the expression of long non-coding RNA gene MEG3 in HCC [29, 30]. Therefore, miRNAs represent a promising new family of targets in the current era of molecular therapies in oncology [31-38]. Genome-wide miRNA expression profiling showed that miR-26a dysregulation in diverse diseases. MiR-26a is located in 3p23, which is a fragile chromosomal region associated with various human cancers [39-47]. In spite of some studies have shown that it functions as an oncogene in cancers like Ovarian Cancer and Lung cancer $[42,43]$, emerging evidence demonstrated that miR-26a may serve as a potential 


\section{Cellular Physiology \\ Cell Physiol Biochem 2017;41:583-597

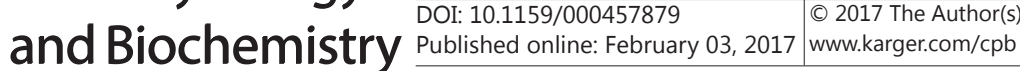

Fig. 8. MiR-26a/EZH2-mediated signal pathway inhibited tumor growth and EMT in vivo. SPC-A1/DTX cells were transfected with miR-26a or co-transfected with pcDNA3.1/EZH2 and injected subcutaneously into nude mice. (A) Growth curve of tumor volumes and representative photographs of tumor bearing mouse and tumors formed 28 days. (B) Western blotting was utilized to detect the expression level of epithelial markers (E-cadherin, $\beta$-catenin) and the mesenchymal markers (N-cadherin, vimentin). (C) Immunostaining of Ki-67 and PCNA protein of the transplanted tumors as indicated (original magnification, $\times 400$ ). (D-E) TUNEL assay stained and western blotting assay were employed to detect the apoptosis rate of tissues from transplanted tumors. Error bars represent the mean \pm SEM of at least three independent experiments. ${ }^{*} p<0.05,{ }^{* *} p<0.01$ vs. control group.

tumor suppressor in other malignant tumors [46, 48]. In 2014, Jiang DS et.al demonstrated that miR-26 is involved in the TLR9-mediated growth and migration of lung cancer through PI3K/Akt signaling pathway [13]. Additional, in 2016, Yang Y et.al revealed that decreased miR-26a expression could lead to cisplatin resistance in human non-small cells lung cancer through regulating HMGA1-mediated E2F1-Akt pathway [14]. Given the function of miR-26a reported in lung cancer, we hypothesis that miR-26a might be involved in the malignant phenotype acquisition of docetaxel-resistant LAD cells.

To gain insight of the biological function of miR-26a in docetaxel-resistant LAD cells, we first employed qRT-PCR assays to evaluate the expression level of miR-26a in docetaxelresistant LAD cells and the parental cells and showed that the expression level of miR-26a in docetaxel-resistant LAD cells was significantly lower than that in parental cells. Then, function assays confirmed that forced expression of miR-26a could obviously suppress the proliferation, facilitate the apoptosis, inhibit the metastasis ability and reverse the EMT to MET of docetaxel-resistant LAD cells. It was proposed that miR-26a may show critical roles in the progression of docetaxel-resistant LAD cells.

EZH2 is a critical component of the polycomb-repressive complex2 (PRC2) which contribute to many essential biological processes. The oncogenic role of EZH2 has recently been documented in several types of cancer [18, 19, 49]. MiR-26a has been reported to suppress tumorgenesis by targeting EZH2 in several tumors [24, 50]. To explore whether EZH2 involved in the miR-26a-mediated function in docetaxel-resistant LAD cells, we detected the expression level of EZH2 in docetaxel-resistant LAD cells and parental cells. Then, qRT-PCR and western blot assays were employed to exam the function of miR-26a on expression of EZH2. Consistent to our assumption, EZH2 is a downstream target of miR-26a and overexpression of miR-26 could remarkably decrease the level of EZH2 in both mRNA and protein level. Further luciferase assay confirmed the relationship between miR-26a and EZH2. To further investigate the role of EZH2 in docetaxel-resistant LAD cells, function assays were performed and results showed that downexpression of EZH2 could significantly suppress the proliferation, facilitate the apoptosis, inhibit the metastasis ability and reverse the EMT to MET of docetaxel-resistant LAD cells. To make a further confirm, rescue assays 


\section{Cellular Physiology Cell Physiol Biochem 2017;41:583-597

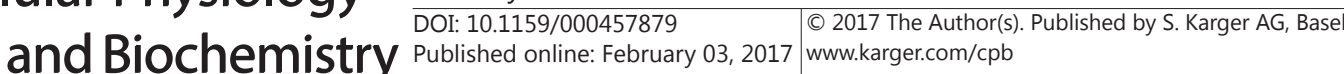 \\ Chen et al:: MiR-26a Regulates the LAD Through EZH2}

were employed. All the data together indicates that EZH2 may involve in the miR-26amediated function in docetaxel-resistant LAD cells.

The present study revealed that miR-26a was significantly down-regulated in the dcetaxel-insensitive groups $(n=19)$ compared with the docetaxel-sensitive groups $(n=18)$. Overexpression of miR-26a could significantly suppress the proliferation, facilitate the apoptosis, cause G2/M phrase arrest, inhibit the metastasis ability and reverse the EMT to MET of docetaxel-resistant LAD cells. Our data suggest that the function of miR-26a in LAD cells is partially exerted via downregulating EZH2. Summary, we first revealed that miR-26a/ EZH2 signal pathway makes contribute to the malignant phenotype of docetaxel-resistant of LAD cells.

\section{Conclusion}

In conclusion, our study demonstrated that miR-26a function as tumor suppressor through directly target EZH2 in the progression of LAD. These findings provided a new insight into the molecular pathogenesis of chemoresistant-LAD.

\section{Abbreviations}

EZH2 (enhancer of zeste homolog 2); EMT (epithelial-to-mesenchymal); H\&E (hematoxylin and eosin); HMGA2 (High mobility group A2); LAD (lung adenocarcinoma); MEG3 (maternally expressed gene 3); PCNA (proliferating cell nuclear antigen); PRC2 (polycomb-repressive complex2); SDS-PAGE (sodium dodecyl suifate-polyacrylamide gel electrophoresis); siRNA (small interfering RNA); TUNEL (terminal-deoxynucleoitidyl Transferase Mediated Nick End Labeling assay).

\section{Acknowledgements}

This study was sponsored by the National Science Foundation of China (81272474) and (81572933).

\section{Disclosure Statement}

The authors declare that they have no competing interests.

\section{References}

$\rightarrow 1$ Siegel RL, Miller KD, Jemal A: Cancer Statistics, 2016. CA Cancer J Clin 2016;66:7-30.

- Liu S, Tang J, Huang L, Xu Q, Ling X, Liu J: Cordyceps Militaris Alleviates Severity of Murine Acute Lung Injury Through miRNAs-Mediated CXCR2 Inhibition. Cell Physiol Biochem 2015;36:2003-2011.

-3 Gettinger S, Lynch T: A Decade of Advances in Treatment for Advanced Non-Small Cell Lung Cancer. Clin Chest Med 2011;32:839-51.

4 Cai M, Wang Z, Zhang J, Zhou H, Jin L, Bai R, Weng Y: Adam17, a Target of Mir-326, Promotes Emt-Induced Cells Invasion in Lung Adenocarcinoma. Cell Physiol Biochem 2015;36:1175-1185.

$>5$ Deng W, Yan M, Yu T, Ge H, Lin H, Li J, Liu Y, Geng Q, Zhu M, Liu L, He X, Yao M: Quantitative proteomic analysis of the metastasis-inhibitory mechanism of miR-193a-3p in non-small cell lung cancer. Cell Physiol Biochem 2015;35:1677-1688.

6 Tang B, Lei B, Qi G, Liang X, Tang F, Yuan S, Wang Z, Yu S, He S: MicroRNA-155-3p promotes hepatocellular carcinoma formation by suppressing FBXW7 expression. J Exp Clin Cancer Res 2016;35:93.

7 Wu G, Zheng K, Xia S, Wang Y, Meng X, Qin X, Cheng Y: MicroRNA-655-3p functions as a tumor suppressor by regulating ADAM10 and beta-catenin pathway in Hepatocellular Carcinoma. J Exp Clin Cancer Res 2016;35:89.

-8 Chen W, Wang J, Liu S, Wang S, Cheng Y, Zhou W, Duan C, Zhang C: MicroRNA-361-3p suppresses tumor cell proliferation and metastasis by directly targeting SH2B1 in NSCLC. J Exp Clin Cancer Res 2016;35:76. 


\section{Cellular Physiology Cell Physiol Biochem 2017;41:583-597 and Biochemistry Poblished online: February 03, 2017 www.karger.com/cpb}

Chen et al:: MiR-26a Regulates the LAD Through EZH2

9 Chen Y, Gao Y, Zhang K, Li C, Pan Y, Chen J, Wang R, Chen L: MicroRNAs as Regulators of Cisplatin Resistance in Lung Cancer. Cell Physiol Biochem 2015;37:1869-1880.

-10 Li J, Yu T, Cao J, Liu L, Liu Y, Kong HW, Zhu MX, Lin HC, Chu DD, Yao M, Yan MX: MicroRNA-148a Suppresses Invasion and Metastasis of Human Non-Small-Cell Lung Cancer. Cell Physiol Biochem 2015;37:1847-1856.

11 Zhu X, Fu C, Zhang L, Xu G, Wang S: MiRNAs associated polymorphisms in the 3'UTR of MET promote the risk of non-small cell lung cancer. Cell Physiol Biochem 2015;37:1159-1167.

12 Liu H, Chen M, Wu F, Li F, Yin T, Cheng H, Li W, Liu B, Wang Q, Tao L: rs2910164 Polymorphism Confers a Decreased Risk for Pulmonary Hypertension by Compromising the Processing of microRNA-146a. Cell Physiol Biochem 2015;36:1951-1960.

13 Jiang D-S, Wang Y-W, Jiang J, Li S-M, Liang S-Z, Fang H-Y: MicroRNA-26a involved in Toll-like receptor 9-mediated lung cancer growth and migration. Int J Mol Med 2014;34:307-312.

14 Yang Y, Zhang P, Zhao Y, Yang J, Jiang G, Fan J: Decreased MicroRNA-26a expression causes cisplatin resistance in human non-small cell lung cancer. Cancer Biol Ther 2016;17:515-525.

15 Ma DN, Chai ZT, Zhu XD, Zhang N, Zhan DH, Ye BG, Wang CH, Qin CD, Zhao YM, Zhu WP, Cao MQ Gao DM, Sun HC, Tang ZY: MicroRNA-26a suppresses epithelial-mesenchymal transition in human hepatocellular carcinoma by repressing enhancer of zeste homolog 2. J Hematol Oncol 2016;9:1.

16 Zhu Z, Tang J, Wang J, Duan G, Zhou L, Zhou X: MiR-138 Acts as a Tumor Suppressor by Targeting EZH2 and Enhances Cisplatin-Induced Apoptosis in Osteosarcoma Cells. PLoS One 2016;11:e0150026.

17 Marchesi I, Bagella L: Targeting Enhancer of Zeste Homolog 2 as a promising strategy for cancer treatment. World J Clin Oncol 2016;7:135-148.

18 Chen W-M, Huang MD, Sun D-P, Kong R, Xu T-P, Xia R, Zhang E-B, Shu Y-Q: Long intergenic non-coding RNA 00152 promotes tumor cell cycle progression by binding to EZH2 and repressing p15 and p21 in gastric cancer. Oncotarget 2016;7:9773-9787.

19 Jang S-H, Lee JE, Oh M-H, Lee J-H, Cho HD, Kim K-J, Kim SY, Han SW, Kim HJ, Bae SB, Lee HJ: High EZH2 Protein Expression Is Associated with Poor Overall Survival in Patients with Luminal A Breast Cancer. J Breast Cancer 2016;19:53-60.

20 Yu W, Zhang G, Lu B, Li J, Wu Z, Ma H, Wang H, Lian R: miR-340 impedes the progression of laryngeal squamous cell carcinoma by targeting EZH2. Gene 2016;577:193-201.

-21 Riquelme E, Behrens C, Lin HY, Simon G, Papadimitrakopoulou V, Izzo J, Moran C, Kalhor N, Lee JJ, Minna JD, Wistuba II: Modulation of EZH2 Expression by MEK-ERK or PI3K-AKT Signaling in Lung Cancer Is Dictated by Different KRAS Oncogene Mutations. Cancer Res 2016;76:675-685.

22 Geng J, Li X, Zhou Z, Wu C-L, Bai X, Dai M: EZH2 promotes tumor progression via regulating VEGF-A/AKT signaling in non-small cell lung cancer (vol 359, pg 275, 2015). Cancer Lett 2016;370:366-366.

23 Jansen MPHM, Reijm EA, Sieuwerts AM, Ruigrok-Ritstier K, Look MP, Rodriguez-Gonzalez FG, Heine AAJ, Martens JW, Sleijfer S, Foekens JA, Berns EMJJ: High miR-26a and low CDC2 levels associate with decreased EZH2 expression and with favorable outcome on tamoxifen in metastatic breast cancer. Breast Cancer Res Treat 2012;133:937-947.

24 Ma D-N, Chai Z-T, Zhu X-D, Zhang N, Zhan D-H, Ye B-G, Wang C-H, Qin C-D, Zhao Y-M, Zhu W-P, Cao M-Q Gao D-M, Sun H-C, Tang Z-Y: MicroRNA-26a suppresses epithelial-mesenchymal transition in human hepatocellular carcinoma by repressing enhancer of zeste homolog 2. J Hematol Oncol 2016;9:1.

25 Zhuang C, Wang P, Huang D, Xu L, Wang X, Wang L, Hu L: A double-negative feedback loop between EZH2 and miR-26a regulates tumor cell growth in hepatocellular carcinoma. Int J Oncol 2016;48:1195-1204.

-26 Zhu Z, Tang J, Wang J, Duan G, Zhou L, Zhou X: MiR-138 Acts as a Tumor Suppressor by Targeting EZH2 and Enhances Cisplatin-Induced Apoptosis in Osteosarcoma Cells. Plos One 2016;11:e0150026.

27 Wang C, Liu X, Chen Z, Huang H, Jin Y, Kolokythas A, Wang A, Dai Y, Wong DTW, Zhou X: Polycomb group protein EZH2-mediated E-cadherin repression promotes metastasis of oral tongue squamous cell carcinoma. Mol Carcinog 2013;52:229-236.

28 Wu Y, Zhang L, Zhang L, Wang Y, Li H, Ren X, Wei F, Yu W, Liu T, Wang X, Zhou X, Yu J, Hao X: Long noncoding RNA HOTAIR promotes tumor cell invasion and metastasis by recruiting EZH2 and repressing E-cadherin in oral squamous cell carcinoma. Int J Oncol 2015;46:2586-2594.

29 Han H-B, Gu J, Zuo H-J, Chen Z-G, Zhao W, Li M, Ji D-B, Lu Y-Y, Zhang Z-Q: Let-7c functions as a metastasis suppressor by targeting MMP11 and PBX3 in colorectal cancer. J Pathol 2012;226:544-555. 


\section{Cellular Physiology \\ Cell Physiol Biochem 2017;41:583-597

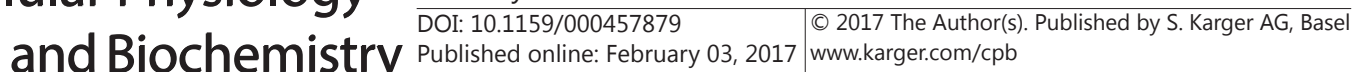

Chen et al.: MiR-26a Regulates the LAD Through EZH2

-30 Braconi C, Kogure T, Valeri N, Huang N, Nuovo G, Costinean S, Negrini M, Miotto E, Croce CM, Patel T: microRNA-29 can regulate expression of the long non-coding RNA gene MEG3 in hepatocellular cancer. Oncogene 2011;30:4750-4756.

31 He Z, Xia Y, Pan C, Ma T, Liu B, Wang J, Chen L, Chen Y: Up-Regulation of MiR-452 Inhibits Metastasis of NonSmall Cell Lung Cancer by Regulating BMI1. Cell Physiol Biochem 2015;37:387-398.

32 Huang C, Ma R, Yue J, Li N, Li Z, Qi D: MiR-497 Suppresses YAP1 and Inhibits Tumor Growth in Non-Small Cell Lung Cancer. Cell Physiol Biochem 2015;37:342-352.

-33 Yan A, Yang C, Chen Z, Li C, Cai L: MiR-761 Promotes Progression and Metastasis of Non-Small Cell Lung Cancer by Targeting ING4 and TIMP2. Cell Physiol Biochem 2015;37:55-66.

-34 Chen S, Li P, Li J, Wang Y, Du Y, Chen X, Zang W, Wang H, Chu H, Zhao G, Zhang G: MiR-144 inhibits proliferation and induces apoptosis and autophagy in lung cancer cells by targeting TIGAR. Cell Physiol Biochem 2015;35:997-1007.

-35 Wan L, Zhu L, Xu J, Lu B, Yang Y, Liu F, Wang Z: MicroRNA-409-3p functions as a tumor suppressor in human lung adenocarcinoma by targeting c-Met. Cell Physiol Biochem 2014;34:1273-1290.

36 Zhang L, Qian J, Qiang Y, Huang H, Wang C, Li D, Xu B: Down-regulation of miR-4500 promoted non-small cell lung cancer growth. Cell Physiol Biochem 2014;34:1166-1174.

-37 Hu X, Zhang F, Liu XR, Wu YT, Ni YM: Efficacy and potential microRNA mechanism for computed tomography-guided percutaneous radiofrequency ablation of primary lung cancer and lung metastasis from liver cancer. Cell Physiol Biochem 2014;33:1261-1271.

38 Wu N, Zhang C, Bai C, Han YP, Li Q: MiR-4782-3p inhibited non-small cell lung cancer growth via USP14. Cell Physiol Biochem 2014;33:457-467.

-39 Wen L, Cheng F, Zhou Y, Yin C: MiR-26a Enhances the Sensitivity of Gastric Cancer Cells to Cisplatin by Targeting NRAS and E2F2. Saudi J Gastroenterol 2015;21:313-319.

-40 Wang G, Sun Y, He Y, Ji C, Hu B, Sun Y: miR-26a Promoted by Interferon-Alpha Inhibits Hepatocellular Carcinoma Proliferation and Migration by Blocking EZH2. Genet Test Mol Biomarkers 2015;19:30-36.

41 Wang L-J, Zhang K-L, Zhang N, Ma X-W, Yan S-W, Cao D-H, Shi S-J: Serum miR-26a as a diagnostic and prognostic biomarker in cholangiocarcinoma. Oncotarget 2015;6:18631-18640.

42 Liu B, Wu X, Liu B, Wang C, Liu Y, Zhou Q Xu K: MiR-26a enhances metastasis potential of lung cancer cells via AKT pathway by targeting PTEN. Biochim Biophys Acta 2012;1822:1692-1704.

-43 Shen W, Song M, Liu J, Qiu G, Li T, Hu Y, Liu H: MiR-26a Promotes Ovarian Cancer Proliferation and Tumorigenesis. Plos One 2014;9:e86871.

44 Fu X, Meng Z, Liang W, Tian Y, Wang X, Han W, Lou G, Wang X, Lou F, Yen Y, Yu H, Jove R, Huang W: miR-26a enhances miRNA biogenesis by targeting Lin28B and Zcchc11 to suppress tumor growth and metastasis. Oncogene 2014;33:4296-4306.

-45 Li X, Lian L, Zhang D, Qu L, Yang N: gga-miR-26a targets NEK6 and suppresses Marek's disease lymphoma cell proliferation. Poult Sci 2014;93:1097-1105.

46 Liu P, Tang H, Chen B, He Z, Deng M, Wu M, Liu X, Yang L, Ye F, Xie X: miR-26a suppresses tumour proliferation and metastasis by targeting metadherin in triple negative breast cancer. Cancer Lett 2015;357:384-392.

47 Wang S, Dong W, Xie J, He L, Zhou X, Cai Y, Liu J, Xie W: miR-26a inhibits cell proliferation by regulating TFAP2C expression in ovarian cancer cells. Tumor 2014;34:908-912,956.

-48 Lu J, He M-L, Wang L, Chen Y, Liu X, Dong Q, Chen Y-C, Peng Y, Yao K-T, Kung H-F, Li X-P: MiR-26a Inhibits Cell Growth and Tumorigenesis of Nasopharyngeal Carcinoma through Repression of EZH2. Cancer Res 2011;71:225-233.

49 Smonskey M, Lasorsa E, Rosario S, Kirk JS, Hernandez-Ilizaliturri FJ, Ellis L: EZH2 inhibition re-sensitizes multidrug resistant B-cell lymphomas to etoposide mediated apoptosis. Oncoscience 2016;3:21-30.

50 Yu L, Lu J, Zhang B, Liu X, Wang L, Li S-Y, Peng X-H, Xu X, Tian W-D, Li X-P: miR-26a inhibits invasion and metastasis of nasopharyngeal cancer by targeting EZH2. Oncol Lett 2013;5:1223-1228. 